DIVISION OF THE HUMANITIES AND SOCIAL SCIENCES

CALIFORNIA INSTITUTE OF TECHNOLOGY

PASADENA, CALIFORNIA 91125

THE APPROXIMATION OF EFFICIENT PUBLIC GOOD MECHANISM BY SIMPLE VOTING SCHEMES

John O. Ledyard

Thomas R. Palfrey

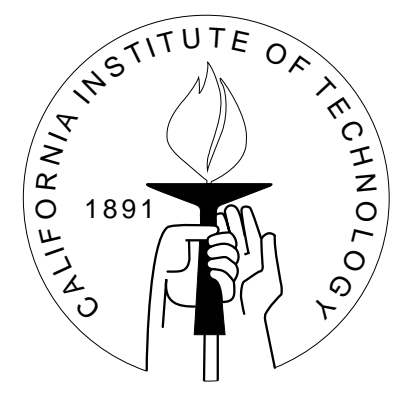

SOCIAL SCIENCE WORKING PAPER 1092R

August 1999

Revised October 2000 


\title{
The Approximation of Efficient Public Good Mechanisms by Simple Voting Schemes*
}

\author{
John O. Ledyard \\ California Institute of Technology \\ Thomas R. Palfrey ${ }^{\dagger}$ \\ California Institute of Technology
}

October 12,2000

\begin{abstract}
This paper compares the performance of simple voting rules, called referenda, to the performance of interim efficient mechanisms for the provision of a public good. In a referendum, voters simply vote for or against the provision of the public good, and production of the public good depends on whether or not the number of yes votes exceeds a prespecified threshold. Costs are shared equally. We show that in large populations for any interim efficient allocation rule, there exists a corresponding referendum that yields approximately the same total welfare when there are many individuals. Moreover, if there is a common value component to the voters' preferences, then there is a unique approximating referendum.

JEL Classification numbers: D61, D82, H41 Key words: public goods; interim efficiency; voting; simple mechanisms

*This is a revision of a paper entitled "Voting is Approximately Optimal." We are grateful for the support of the National Science Foundation and of the New Millennium Program of the Jet Propulsion Laboratory of NASA. The second author is grateful for the hospitality and research support at LEI-CREST. We thank two anonymous referees for their comments.

${ }^{\dagger}$ (corresponding author) Division of Humanities and Social Sciences, Mail Code 228-77, California Institute of Technology, Pasadena, CA 91125. trp@hss.caltech.edu Fax:(626)432-1726. Phone:
\end{abstract} (626)395-4088. 


\section{Introduction}

In his seminal paper on decentralization, Hurwicz (1972) sets out a general framework for the mechanism design approach to welfare economics. Many subsequent papers have followed through on the insights of that paper in various applications, including auctions, contracting, public goods, organizational structure, and other topics. Yet one important component of the Hurwicz framework is absent from most of this work. That component is the cost of operating the mechanism. Instead, the primary focus has been on the constraints imposed by incentive compatibility and individual rationality. The tradeoff we wish to focus on in this paper is between the attainment of an incentive-constrained optimum and the cost of the institution required to do so. The closest attempts to explore this issue have posed the question, "What is the smallest message space required to exactly implement the optimum?" ${ }^{1}$ While this does indirectly address the issue of how costly it is to decentralize certain outcomes, it does not ask the tradeoff question: "When is it better to use a cheaper mechanism and attain somewhat less than the incentive-constrained optimum?" This paper addresses a version of the latter question in the context of a classical public goods environment.

A group of individuals must decide on a level of a public good that is produced according to constant returns to scale up to some capacity constraint. In addition to deciding the level of public good, the group must decide how to tax the individuals in the group in order to cover the cost. The distribution of the burden of taxation is

\footnotetext{
${ }^{1}$ The seminal paper is Mount and Reiter (1974). The problem has also been investigated in the context of team theory. See, for example, Marschak and Radner (1972).
} 
important because different individuals have different marginal rates of substitution between the private good (taxes) and the public good. These individual marginal rates of substitution are private information; that is, each individual knows his or her own marginal rate of substitution, but not those of the other members of the group. Adopting a Bayesian mechanism design framework, we assume that the distribution of marginal rates of substitution is common knowledge.

We are interested in characterizing the performance of simple mechanisms with extremely small message spaces, and comparing performance with optimal mechanisms, using interim efficiency as the benchmark. In an earlier paper (Ledyard and Palfrey 1999), we fully characterized the set of interim efficient mechanisms, and we use this characterization in the analysis. Interim efficiency is the natural extension of Pareto optimality when applied to problems of Bayesian mechanism design. An allocation rule is interim efficient if it is (Bayesian) incentive compatible and there is no other Bayesian incentive compatible allocation rule that is (weakly) prefered by all types of all agents. In the context of our public good problem, the classical LindahlSamuelson public good decision (coupled with particular incentive taxes) is but one of many interim efficient allocation rules. In fact, there is an infinite dimensional set of interim efficient public good allocation rules, which vary with respect to both the incidence of incentive taxes across agents and types and also the mapping from type profiles to the public good decision.

The simple mechanisms we investigate are referenda, which are the class of anonymous and informative mechanisms that use the smallest possible message space. Each player's message contains one bit of information, which we call a vote. If the proportion of yes voters exceeds a prespecified threshold, then the public good is produced 
at maximum capacity and costs are shared equally among all agents. If the proportion of yes voters falls short of the prespecified threshold, then the public good is not produced and no taxes are collected.

An earlier paper establishes the efficiency of referenda and equal taxation for the very special case where types are identically distributed and can only take on two values (Ledyard and Palfrey 1994). There it was shown that interim efficiency always takes a special form whereby the public good is provided if and only if the number of high valuation types exceeds a threshold number which depends on the welfare weights and the distribution of types. The greater the welfare weight on high valuation types, the lower the optimal threshold. Obviously, such allocation rules require only a single bit of information from the agents, so that a yes/no voting mechanism is isomorphic to an anonymous direct mechanism. With more than two types (as in this paper) the optimal mechanism generally depends on the exact profile of types in a complicated way.

The central finding of this paper is that with large numbers of individuals, a similar result obtains regardless of the number of types. Specifically, we show for a continuum of types that in large populations the performance of the optimal mechanisms can be approximated by referenda. For every interim efficient mechanism there is a referendum such that the aggregate welfare achieved from the voting scheme converges, as the population grows, to the aggregate welfare achieved from the interim efficient mechanism. Moreover, if the distribution of valuations is not known precisely by the planner, then the optimal referendum is uniquely determined.

Therefore, referenda satisfy three important desiderata: efficiency, simplicity, and robustness. It is simple not only with respect to the very small message space, but 
also by avoiding complex tax/transfer rules that depend on the exact profile of types in very complicated ways (d'Aspremont and Gerard-Varet 1979). In addition to their simplicity, these voting mechanisms have the property that players have dominant strategies, which eliminates strategic uncertainty from the points of view of the participants and the planner. Third, referenda are robust with respect to misspecification of prior beliefs or attitudes toward risk. That is, risk averse participants will behave the same as risk neutral participants, and all participants will vote the same regardless of their prior beliefs about the distribution of the other participants' valuations. $^{2}$

Given these attractive properties, it should not be surprising that referenda are so widely used in practice. Many countries rely heavily on these binary mechanisms to decide whether to undertake certain public projects. Examples are abundant. In many states in the US, such as California, bond issues for roads, prisons, schools, and other public projects are often ballot items in general elections, as yes/no initiative about social policy. In Switzerland, national referenda are the rule rather than the exception, to decide many policy issues.

Other papers have examined the behavior of both majority rule and optimal mechanisms for large populations. Mailath and Postlewaite (1990) show that in the limit the only optimal mechanism that satisfies individual rationality never produces the public good. Ledyard and Palfrey (1994) provides an intuition for that result based on

\footnotetext{
${ }^{2}$ This contrasts sharply with previous work that has investigated the possibility of efficient dominant strategy mechanisms in Bayesian environments by Mookherjee and Reichelstein (1992). The dominant strategy mechanisms constructed in that paper are very similar to the original Bayesian incentive compatible mechanisms. They require the use of tax/transfer rules that are as complex as the Bayesian incentive compatible mechanisms, and also require risk neutrality and a correct specification of common prior beliefs.
} 
the fact that as $N \rightarrow \infty$, an individual's "influence", or ability to affect the decision goes to zero, so that all feasible incentive compatible, individually rational mechanisms converge to zero production in the limit. Al-Najjar and Smorodinsky (2000) extend and generalize these and related results about asymptotic influence in voting models.

The remainder of the paper is organized as follows. The basic model is outlined in section 2 and the characterization of interim efficiency is summarized. Section 3 establishes the approximate effiicency of referenda for large populations. Section 4 shows that optimal referenda are unique if there is a random shift parameter to the valuations.

\section{The Model}

There are $N$ people who must decide on the quantity, $q$ of a public good that is produced according to constant returns to scale and has a maximum level $Y=1$. The cost of producing $q \in[0,1]$ is equal to $K q$. In addition, it must be decided how to distribute the production costs. Because of the linear production technology, the optimal level of the public good will always be either 0 or 1, so our results also apply to the case of deciding on whether or not to produce a discrete public good. ${ }^{3}$ We let $a^{i}$ denote individuals $i$ 's share of the cost, in units of the consumption of the private good, and assume it can take any real value. Therefore the set of feasible levels of production and cost shares are given by

\footnotetext{
${ }^{3}$ There are are also knife-edge cases where both 0 and 1 are optimal, in which case any level of the public good between 0 and 1 is also optimal.
} 


$$
\left(a^{1}, \ldots, a^{N}, q\right) \in \Re^{N} \times[0,1]
$$

such that

$$
K q \leq \sum_{i=1}^{N} a^{i} .
$$

Individual preferences are assumed to be risk-neutral and quasilinear in the level of public good production and the taxes (cost shares), so the utility to type $v^{i}$ of agent $i$ for an allocation $(q, a)$ is given by

$$
V^{i}=v^{i} q-a^{i}
$$

Thus, $v^{i}$ represents the marginal rate of substitution between the public and private good, or "public good valuation" of type $v^{i}$. We refer to $v^{i}$ as player $i$ 's "value." We assume that each individual knows his own value, $v^{i}$, and does not know the values of the other individuals. We assume that the individual values $\left(v^{i}\right)$ are independently distributed, with the (common knowledge) $c d f$ of $i$ 's value denoted $F_{i}(\cdot)$ and the support of $F_{i}$ is $V^{i}=\left[\underline{v}^{i}, \bar{v}^{i}\right]$, where $\underline{v}^{i}<K / N<\bar{v}^{i}$. We assume $F_{i}$ has a continuous positive density on $V^{i}$.

\subsection{Mechanisms}

A mechanism is a game form, which consists of a message (strategy) space for each agent and an outcome function mapping message profiles into probability distributions 
over the set of feasible allocations. By the revelation principle, the properties (in terms of allocation rules) of any optimal mechanism can be duplicated by an incentive compatible, direct mechanism in which the message space for agent $i$ is simply the set of possible types (values) in the support of $F_{i}$. A strategy for $i$ in a direct mechanism is a mapping $\sigma_{i}: V^{i} \rightarrow V^{i}$, that is, a decision rule that specifies a reported type for each possible type. We refer to the identity mapping as the truthful strategy. By the linearity of the individual utility functions, there is also no loss in restricting attention to deterministic mechanisms. Thus, we denote a direct mechanism simply as a feasible deterministic allocation rule:

$$
\eta: V^{N} \longrightarrow\left\{\left(a^{1}, \ldots, a^{N}, q\right) \in R^{N} \times[0,1] \mid \sum_{i=1}^{N} a^{i} \geq K q\right\}
$$

We denote the public good allocation component of $\eta$ at type profile $v$ by $q(v)$, and the private good tax for $i$ by $a_{i}(v)$.

Besides feasibility, the main restriction on $\eta$ is that it be incentive compatible, which means that it is a Bayesian equilibrium of $\eta$ for all agents to adopt a strategy of truthfully reporting their type. Given a strategy $\sigma_{i}$, and a mechanism, $\eta$, let the interim utility of type $v^{i}$ of agent $i$, assuming all others truthfully report their type, be denoted by:

$$
\hat{u}_{i}\left(\eta, v^{i}, \sigma^{i}\right)=\int_{V^{-i}}\left[v^{i} q\left(\sigma^{i}\left(v^{i}\right), v^{-i}\right)-a^{i}\left(\sigma^{i}\left(v^{i}\right), v^{-i}\right)\right] d F\left(v \mid v^{i}\right)
$$

Let $u_{i}\left(\eta, v^{i}\right) \equiv \hat{u}_{i}\left(\eta, v^{i}, I\right)$ where $I$ denotes the truthful strategy $I\left(v^{i}\right)=v^{i}$. Then $\eta$ is incentive compatible if and only if $u_{i}\left(\eta, v^{i}\right) \geq \hat{u}_{i}\left(\eta, v^{i}, \sigma_{i}\right)$ for all $v^{i}, \sigma_{i}$. 


\subsection{Optimal Mechanisms}

The set of interim incentive efficient allocation $\operatorname{rules}^{4}$ can be represented as the solutions to a family of maximization problems. Let $\lambda>0$ be a system of welfare weights, a measurable function mapping types into the positive real line, so that $\lambda_{i}\left(v^{i}\right)$ represents the welfare weight assigned to type $v^{i}$ of agent $i$. Then $\eta$ is interim efficient if and only if there exists $\lambda$ such that $\eta$ maximizes $\Sigma_{i} \int_{\underline{v}^{i}}^{\bar{v}^{i}} \lambda_{i}\left(v^{i}\right) u_{i}\left(\eta, v^{i}\right) d F_{i}\left(v^{i}\right)$ over the set of feasible and incentive compatible mechanisms. ${ }^{5}$

Ledyard and Palfrey (1999) characterize the set of interim efficient allocation rules, which can be summarized as follows. Fix a system of welfare weights such that $\int_{\underline{v}^{i}}^{\bar{v}^{i}} \lambda_{i}\left(v^{i}\right) d F_{i}\left(v^{i}\right)=1$. Let

$$
w_{i}\left(v^{i} ; \lambda\right) \equiv v^{i}-\frac{1-F_{i}\left(v^{i}\right)}{f_{i}\left(v^{i}\right)}+\frac{\int_{v^{i}}^{\bar{v}^{i}} \lambda_{i}\left(t^{i}\right) d F_{i}\left(t^{i}\right)}{f_{i}\left(v^{i}\right)}
$$

be called the $\lambda$-virtual valuation of type $v^{i}$ and let $\widehat{q}(v ; \lambda)$ be given by the standard ex post efficiency criterion applied to the individuals' $\lambda$-virtual valuations, $w(v ; \lambda)$. That is, $\widehat{q}(v ; \lambda)$ solves

$$
\max _{q}\left[\left(\sum_{i} w_{i}\left(v^{i}, \lambda\right)\right)-K\right] q
$$

subject to $q \in[0,1]$. Let $\widehat{Q}_{i}\left(v^{i}, \lambda\right)=\int \widehat{q}(v ; \lambda) d F_{-i}\left(v^{-i}\right)$ denote the conditional (on $v^{i}$ ) expected output of the public good. We call $\widehat{Q}$ the reduced form of $\widehat{q}$. Then as long as $\widehat{Q}_{i}$ is non-decreasing in $v^{i}$ for all $i$, then $\widehat{q}(v ; \lambda)$ is interim efficient and can be supported by type-contingent taxes in the standard way. ${ }^{6}$

\footnotetext{
${ }^{4}$ For the remainder, we simply refer to such allocations as "interim efficient."

${ }^{5}$ See Holmstrom and Myerson (1983)

${ }^{6}$ If $\widehat{Q}$ constructed by this algorithm is non-monotonic, then one can use standard techniques
} 


\subsection{Referendum Mechanisms}

We identify a class of simple mechanisms, each of which uses a minimally informative message space. Each individual transmits only a single binary bit of information, which we call a "vote." It is as if each individual is asked whether or not he would like to have the public good produced. If enough voters say "yes," then the public good is produced and the cost is shared equally. Otherwise the public good is not provided and nobody pays anything. We call such mechanisms referenda with equal cost shares. ${ }^{7}$

To be specific a $J^{*}$-referendum has the following three properties:

(a) Each $i$ votes, $b^{i}$, yes $(=1)$ or no $(=0)$.

(b) The good is produced if $\sum_{i} b^{i} \geq J^{*}$ and is not produced if $\sum b^{i}<J^{*}$.

(c) Each $i$ pays $\frac{K}{N}$ if it is produced and 0 if it is not produced.

Thus, in a $J^{*}$-referendum each individual casts a vote either for or against the production of the public good, which is produced if and only if at least $J^{*}$ "yes" votes are cast, and costs are split equally. For each voter, it is a dominant strategy to vote yes, if and only if $v^{i} \geq K / N$. The incentive compatible direct revelation version of this mechanism is:

to flatten out the non-monotonicities. See Guesnerie and Laffont (1984) for a simple geometric explanation of this technique, which is sometimes referred to as "ironing."

${ }^{7}$ In Ledyard and Palfrey (1994) we used the term lottery draft, since equal cost sharing is equivalent (in expected utility) to randomly selecting, or drafting, $M \leq N$ individuals to contribute an equal $(K / M)$ share to the production of the public good. If the private good space is discrete, randomization of this sort is needed. 


$$
\begin{gathered}
q(v)=1 \text { if } \#\left\{i \mid v^{i} \geq \frac{K}{N}\right\} \geq J^{*} \\
=0 \text { if } \#\left\{i \mid v^{i} \geq \frac{K}{N}\right\}<J^{*} \\
a_{i}(v)=\frac{K}{N} q(v) \forall i
\end{gathered}
$$

The reason for considering such mechanisms is that, as we show below, they are almost interim efficient in large populations. By this, we mean that the efficiency loss from using a referendum instead of an optimal mechanism approaches zero in large populations. The two extreme referenda, corresponding to $J^{*}=0$ (always produce) and $J^{*}=N$ (never produce), are of independent interest and we refer to these as command mechanisms.

\section{Approximate Optimality of Referenda}

It is fairly easy to see that, in small finite populations, referenda are generally not interim efficient, except in extreme cases where the critical level of $J^{*}$ is equal to either 0 or $N$ and production is independent of the realization of the type-profile, $v .^{8}$ But suppose there is a per capita cost to operating a mechanism that is increasing in the size of the message space. And suppose the per capita welfare losses from using referenda instead of optimal mechanisms are negligable in large populations.

\footnotetext{
${ }^{8}$ An example of this arises when $v^{i}$ is distributed on the [1,2] interval for all $i$, and $\frac{K}{N}<1$. In this special case, production is always optimal independent of the actual draws of $v$. So $J^{*}=0$ is efficient. Of course, in this case, there is no need to elicit messages from the agents at all.
} 
Then, according to this criterion of net efficiency, simple voting rules outperform the optimal mechanism. We show below that this is always true if $N$ is sufficiently large. We present three approximation results below. In letting $N$ grow, we permit $K$ to vary with $N$, but keep $k$ fixed, where $k=K(N) / N$. That is, the per capita production costs of the public good are held fixed. We restrict attention to the symmetric case where $V^{i}=V^{j}=V$ for all $i, j, F_{i}(t)=F_{j}(t)$ for all $i, j$, and $t \in V$, and $\lambda_{i}(t)=\lambda_{j}(t)$ for all $i, j$, and $t \in V$.

\subsection{Per Capita Welfare Losses from Referenda}

In this section we show that the per capita welfare of an appropriately chosen referendum converges to the per capita welfare of the optimal mechanisms. The intuition for this is simple. For any given set of welfare weights, $\lambda$, and any $N$, consider the $J_{N}^{*}$-referendum with the property that the expected sum of virtual utilities, if exactly $J_{N}^{*}$ individuals vote for production of the public good, is equal to $k N$. For this voting rule, asymptotically in $N$, the public good will be produced if and only if the average virtual utility is greater than or equal to $k$ which is exactly the rule that determines $q(v)$ in section 2.3. Also, since the interim expected public good production $\left(Q_{i}\left(v^{i}\right)\right)$ is type independent in the limit, incentive compatibility requires that the interim-expected optimal taxes approach equal cost shares as the number of agents goes to infinity. Therefore, in the limit as the number of agents goes to infinity, the $J_{N}^{*}$ referendum generates the same per-capita expected welfare as the optimal mechanism.

Strictly speaking there is one other step in the argument. Remember $\widehat{q}$ is optimal 
if and only if $\widehat{Q}_{i}$ is non-decreasing in $v$ for all $i$. Otherwise $\widehat{q}$ must be appropriately ironed. So let us call $\widehat{q}$, and its supporting taxes, the relaxed optimal mechanism to indicate it is chosen ignoring the constraint that $\widehat{Q}$ be non-decreasing. What we show below is that, as $N \rightarrow \infty$, the $J_{N}^{*}$ referendum generates the same per-capita expected welfare as the relaxed optimal mechanism. But the relaxed optimal mechanism must generate at least as much per-capita expected welfare as the optimal mechanism. Therefore, the $J^{*}$ referendum must generate the same per capita expected welfare as the optimal mechanism as $N \rightarrow \infty$.

Consider a sequence of $J_{N}^{*}$-referenda where $J_{N}^{*}=j^{*} N$ is set ${ }^{9}$ such that the expected total virtual utility, if exactly $j^{*}$ fraction of individuals vote "yes," equals $k N$. Denoting $w^{+}=E[w ; v>k]$ and $w^{-}=E[w ; v<k]$, this requires choosing $j^{*}$ so that $j^{*} w^{+}+\left(1-j^{*}\right) w^{-}=k$. What we do below is to replicate the economy, keeping the distribution of individual types constant and also keeping the per capita cost of producing the public good constant, and compare the per capita surplus using this $j^{*}$ rule to the per capita surplus using the optimal rule, and show that in the limit they are the same.

Theorem: 1 Let $K_{N}=k N$, $k$ fixed. Let $\lambda_{i}\left(v^{i}\right)=\lambda\left(v^{i}\right)$ and $F_{i}\left(v^{i}\right)=F\left(v^{i}\right) \forall i$. Let $j^{*}$ satisfy $j^{*} w^{+}+\left(1-j^{*}\right) w^{-}=k$. As $N \longrightarrow \infty$ the referendum mechanism using $J_{N}^{*}=j^{*} N$ is almost interim-efficient in the sense that it is feasible, incentive

\footnotetext{
${ }^{9}$ Since $N$ is finite, there is generally no exact value of $j^{*}$ satisfying this equality condition. What we mean precisely is that $\left(\left(J_{N}^{*}-1\right) / N\right) E[w ; v>k]+\left(\left(N-J_{N}^{*}+1\right) / N\right) E[w ; v<k] \leq k$ and $\left(\left(J_{N}^{*}+1\right) / N\right) E[w ; v>k]+\left(\left(N-J_{N}^{*}-1\right) / N\right) E[w ; v<k] \geq k$.
} 
compatible, and

$$
\frac{1}{N} \sum_{i} \int_{\underline{v}^{i}}^{\bar{v}^{i}} \lambda\left(v^{i}\right) u_{i}\left(\eta_{N}^{V L}, v^{i}\right) d F\left(v^{i}\right) \rightarrow \frac{1}{N} \sum_{i} \int_{\underline{v}^{i}}^{\bar{v}^{i}} \lambda\left(v^{i}\right) u_{i}\left(\eta_{N}^{o}, v^{i}\right) d F\left(v^{i}\right)
$$

where $\eta_{N}^{V L}$ denotes the $J_{N}^{*}$-referendum mechanism with $N$ individuals and $\eta_{N}^{o}$ denotes the optimal mechanism with $N$ individuals.

Proof: Denote by "Y," the number of yes votes. By construction of $j^{*}, E\left[\sum_{i} w_{i} / N\right.$ | "Y" $\left.Y^{*} j^{*} N\right]=k$, so that if there are at least $j^{*} N$ votes, then the expected sum of virtual benefits is greater than or equal to $k N$. As $N \rightarrow \infty$, by the strong law of large numbers, the expected average virtual benefit when exactly $j^{*}$ fraction of the voters vote "Yes" will converge in probability to $k$. In other words, the probability that this $J_{N}^{*}$ rule and the optimal rule make different production decisions for the same profile of types approaches 0 in the limit.

The reduced forms public good decision for the $j^{*}$-referendum mechanism is:

$$
Q_{N}^{V L}\left(v^{i}\right)=\int_{V^{-i}} q_{N}^{V L}(v) d F_{-i}\left(v^{-i}\right)=\operatorname{Prob}\left\{\sum_{l \neq i} b^{l}\left(v^{l}\right) / N \geq j^{*}-\left(b^{i}\left(v^{i}\right) / N\right)\right\}
$$

where

$$
b^{l}\left(v^{l}\right)=\begin{aligned}
& 1 \quad \text { if } v^{l} \geq k \\
& 0 \text { if } v^{l}<k
\end{aligned}
$$

The expected taxes in the $j^{*}$-referendum mechanism are given by:

$$
A_{N}^{V L}\left(v^{i}\right)=\int_{V^{-i}} a_{i N}^{V L}(v) d F_{-i}\left(v^{-i}\right)=k Q_{N}^{V L}\left(v^{i}\right)
$$


In the relaxed optimal mechanism, the reduced form mechanism is given by:

$$
\begin{aligned}
& Q_{N}^{R}\left(v^{i}\right)=\operatorname{Prob}\left\{\sum_{l \neq i} w_{l}\left(v^{l}\right) / N \geq k-\left(w_{i}\left(v^{i}\right) / N\right)\right\} ; \text { and } \\
& A_{N}^{R}\left(v^{i}\right)=\int_{V^{-i}} a_{i}^{R}(v) d F\left(v^{-i}\right)=k Q_{N}^{R}\left(v^{i}\right)
\end{aligned}
$$

For large $N, Q_{N}^{V L}\left(v^{i}\right) \approx Q_{N}^{R}\left(v^{i}\right)$ for all $v^{i}$. Incentive compatibility then implies that for large $N, A_{N}^{V L}\left(v^{i}\right) \approx A_{N}^{R}\left(v^{i}\right)$ for all $v^{i}$. Therefore, for large $N$,

$$
\int_{V^{i}} \lambda\left(v^{i}\right) u^{i}\left(\eta_{N}^{V L}, v^{i}\right) d F\left(v^{i}\right) \rightarrow \int_{V^{i}} \lambda\left(v^{i}\right) u^{i}\left(\eta_{N}^{R}, v^{i}\right) d F\left(v^{i}\right)
$$

But we know that

$$
\begin{gathered}
\sum_{i} \int_{V^{i}} \lambda\left(v^{i}\right) u_{i}\left(\eta_{N}^{V L}, v^{i}\right) d F\left(v^{i}\right) \leq \sum_{i} \int_{V^{i}} \lambda\left(v^{i}\right) u_{i}\left(\eta_{N}^{o}, v^{i}\right) d F\left(v^{i}\right) \\
\leq \sum_{i} \int_{V^{i}} \lambda\left(v^{i}\right) u^{i}\left(\eta_{N}^{R}, v^{i}\right) d F\left(v^{i}\right) .
\end{gathered}
$$

Since all individuals are identical, this implies

$$
\frac{1}{N} \sum_{i} \int_{V^{i}} \lambda\left(v^{i}\right) u_{i}\left(\eta_{N}^{V L}, v^{i}\right) d F\left(v^{i}\right) \rightarrow \frac{1}{N} \sum_{i} \int_{V^{i}} \lambda\left(v^{i}\right) u_{i}\left(\eta_{N}^{o}, v^{i}\right) d F\left(v^{i}\right) . \quad \mathrm{QED}
$$

While this is a strong result, as far as justifying the use of simple dominant strategy mechanisms for public good decisions, it would be even nicer to have a stronger result. The reason to look for a stronger result is simple. One can show that in the limit, 
for any optimal public good mechanism, the limit of $Q_{N}$ is either 0 or 1 , depending on the distribution of types and the welfare weights. Thus, using a similar argument as in the proof of the theorem above, one can show that any sequence of voting rules (or any sequence of mechanisms in general), with the property that the expected production of the public good in the limit is the same as the optimal mechanism (either 0 or 1 , respectively), will also generate the same per capita welfare benefits as the optimal mechanism.

Suppose for example that $E[w]>k$. Then the command mechanism "always produce," while being suboptimal for any finite value of $N$ (and worse than the best referendum, as well) generates the same per capita surplus as the optimal mechanism in the limit, since there is almost surely production of $q=1$ in the limit. Moreover, any $j^{*} N$-referendum that fixes $j^{*}$ less than some critical level, is asymptotically optimal. Alternately, suppose that $E[w]<k$. Then the mechanism "never produce," while being suboptimal for any finite value of $N$, generates the same per capita surplus as the optimal mechanism in the limit, since there is almost surely zero production in the limit. Obviously, this indeterminacy problem arises because the planner can compute the optimal limiting production decision ex ante. We next show that this indeterminacy of optimal referenda in the limit is no longer problematic if one considers a more realistic model in which the optimal limiting production decision is not known by the planner ex ante. We obtain below the much stronger result that, if there is ex ante uncertainty about the optimal limiting production decision, then there exists a uniquely optimal referendum rule, which is not a command mechanism. 


\subsection{Total Welfare Losses from $j_{N^{-}}^{*}$ Referenda}

A stronger criterion for asymptotic efficiency is the total (as opposed to per capita) surplus loss of the $j^{*} N$-referendum compared to the optimal mechanism. We prove below that the total surplus loss from using the optimal referendum instead of the optimal virtual cost-benefit criterion goes to zero in the limit. We prove this for the pure private values model (without the common value shift parameter), but it is a straightforward exercise to demonstrate that the same result holds for the more general model with the shift parameter. Moreover, for reasons shown in the previous section, the more general result is uniquely true for the referendum rule defined by $E\left[\sum_{i} w_{i} / N \mid \#\left\{i: v^{i}+c^{*} \geq k\right\}=j^{*}\right]=k-c^{*}$. Since the argument is the same, we omit it here.

By symmetry, the total expected welfare from the optimal mechanism is equal to (suppressing the $i$ superscripts, for convenience):

$$
W_{N}^{o}=N \int_{\underline{v}}^{\bar{v}} \lambda(v)\left[v Q_{N}^{o}(v)-A_{N}^{o}(v)\right] d F(v) .
$$

and the expected welfare from a $j^{*} N$-referendum is:

$$
W_{N}^{j^{*}}=N \int_{\underline{v}}^{\bar{v}} \lambda(v)(v-k) Q_{N}^{j^{*}}(v) d F(v) .
$$

Therefore, the difference in the expected total welfare (i.e., the expected welfare loss) is equal to:

$$
\Delta W_{N}^{j^{*}} \equiv W_{N}^{o}-W_{N}^{j^{*}}
$$


Theorem 2 Let $K_{N}=k N$, $k$ fixed. Let $\lambda_{i}\left(v^{i}\right)=\lambda\left(v^{i}\right)$ and $F_{i}\left(v^{i}\right)=F\left(v^{i}\right), \forall i$. Let $j^{*}$ satisfy $j^{*} w^{+}+\left(1-j^{*}\right) w^{-}=k$. Then the $j^{*} N-$ referendum is asymptotically interim-efficient in the sense that it satisfies $(I)$ and $(F)$ and:

$$
\lim _{N \rightarrow \infty} \Delta W_{N}^{j^{*}} \rightarrow 0
$$

Proof: A complete proof is given in the appendix. An outline of the argument is as follows.

There are two sources of welfare loss from the referendum. First, there is some distortion in the quantity of public good that is produced, since individuals send information only about whether they have "high" or "low" valuations. Fully optimal mechanisms generally require more precise information about the profile of valuations. Second, all types pay equal cost shares in the referendum, although the optimal mechanism typically requires the cost shares to be type-dependent. The proof proceeds by showing that the total welfare losses from each of these two sources goes to zero in the limit.

The magnitude of the first source of inefficiency is on the order of $N$ times the average expected difference in the reduced form production decisions, $Q_{N}^{o}$ and $Q_{N}^{j^{*}}$. Both $Q_{N}^{o}$ and $Q_{N}^{j^{*}}$ are deterministic and equal to each other in the limit (i.e., equal either 0 or 1). Thus, we only need to obtain a rate of convergence to 0 for $Q_{N}^{j^{*}}-Q_{N}^{o}$ and show that this converges to 0 very fast. Specifically, we apply a central limit theorem to show that the speed of convergence is at least on the order of $\sqrt{N} e^{-N}$

The magnitude of the second source of inefficiency is on the order of $N$ times the expected difference between equal cost sharing in the referendum and incentive com- 
patible cost sharing in the optimal mechanism. By incentive compatibility, optimal cost sharing converges to equal cost sharing in the limit. We show that the speed of convergence is at least on the order of $\sqrt{N} e^{-N}$. QED

\section{Common Values: Uniqueness of the Approxi- mately Optimal Referendum}

A simplifying assumption has been made in the above analysis, that the distribution of valuations is known by the planner. This simplification is convenient, but it is the source of an unsatisfying result in the previous section. In particular, if the planner knows exactly the distribution of types, then in the limit the planner also knows the optimal level of production almost surely, so no referendum would needed. In other words, while it is true that referenda are approximately optimal, it is also true that not even a referendum is needed at the limit! We show in this section that a natural and straightforward generalization of the model demonstrates that for any given welfare weighting scheme there is generally a unique referendum rule that is approximately optimal in the limit.

Define $c$ to be a common value component which is added to everyone's private valuation, $v^{i}$, to generate an adjusted individual valuation denoted $r^{i}=v^{i}+c$. Thus $c$ is a parameter which shifts the distribution of valuations either up or down, depending on whether $c$ is positive or negative, respectively. We continue to use the notation as before, where $F(v)$ denotes the cdf of private valuations evaluated at $v$, and $\lambda(v)$ denotes the welfare weight assigned to a $v$-type. The common value com- 
ponent $c$ is distributed according the continuous cdf, $G(c)$. One can easily show that for sufficiently low values of $c$, the public good should never be produced, and for sufficiently high values of $c$, the public good should always be produced. We assume that the support of $G$ is the interval $[\underline{c}, \bar{c}]$ where $\underline{c}<k-\bar{v}$ and $\bar{c}>k-\underline{v}$, so the range of possible common values is large enough to include very high and very low values of $c$, where command mechanisms are optimal. Each individual observes $r^{i}$ and $c$. The planner only knows $F .^{10}$

We now consider optimal referenda under this alternative preference structure, with this common value uncertainty. ${ }^{11}$ Let $\widehat{W}_{N}^{j}$ and $\widehat{W}_{N}^{o}(c)$ denote, respectively, the total surplus under the $j N$ referendum assuming the planner doesn't know $c$, and the total surplus the planner could hypothetically achieve under $\widehat{q}(v ; \lambda)$ if the planner actually knew c. ${ }^{12}$ This is clearly an upper bound on what an optimal mechanism could achieve when the planner does not know $c$. We show below that there exists a unique referendum that achieves this upper bound in the limit. Let $\Delta W_{N}^{j^{*}}=$ $\widehat{W}_{N}^{j^{*}}-\widehat{W}_{N}^{o}(c)$.

Theorem 3 Let $K_{N}=k N$, $k$ fixed. Let $\lambda_{i}\left(v^{i}\right)=\lambda\left(v^{i}\right)$ and $F_{i}\left(v^{i}\right)=F\left(v^{i}\right), \forall i$. Assume $G$ has full support on $[\underline{c}, \bar{c}]$ where $\underline{c}<k-\bar{v}$ and $\bar{c}>k-\underline{v}$. Let $j^{*}$ satisfy $E\left[\sum_{i} w_{i} / N \mid \#\left\{i: v^{i}+c^{*} \geq k\right\}=j^{*}\right]=k-c^{*}$. Then the $j^{*} N-$ referendum is uniquely asymptotically interim-efficient in the sense that it it satisfies (I) and (F) and is the

\footnotetext{
${ }^{10}$ Neither the planner nor the individuals have to know $G$.

${ }^{11}$ Since referenda are dominant strategy mechanisms, it is not actually necessary to specify any particular common knowledge structure for the players, beyond the fact that $r^{i}$ is known by $i$. But for concreteness, we have chosen to assume that $c$ be common knowledge among the individuals and assume $v^{i}$ is private information as before, with common prior beliefs about $v$ given by $F$.

${ }^{12}$ In other words, the benchmark "optimal mechanism" to which the referendum is compared is the solution to a doubly relaxed optimization problem. That is, the optimization assumes the planner knows $c$, and also assumes the constraint that $\widehat{Q}$ be nondecreasing is not imposed.
} 
only $j N$ referendum such that:

$$
\lim _{N \rightarrow \infty} \Delta W_{N}^{j} \rightarrow 0
$$

Proof: It is easy to see that, conditional on $c$, the optimal mechanism requires production if and only if the average virtual valuation exceeds $k-c$. That is, the cost-benefit criterion is simply shifted by the common value parameter. Denote by $c^{*}$ the critical value of $c$ such that $E\left[w\left(v^{i}\right) ; \lambda\right]=k-c^{*}$. That is, if $c>c^{*}$ then the public good should always be produced in the limit, and if $c<c^{*}$ it should never be produced in the limit. Since $\underline{c}<k-\bar{v}$, it must be that $c^{*}>\underline{c}$, and $\bar{c}>k-\underline{v}$ implies that $c^{*}<\bar{c}$. Therefore, $c^{*}$ lies in the interior of the support of $G$. Now consider the $j^{*} N$-referendum defined uniquely ${ }^{13}$ by $E\left[\sum_{i} w_{i} / N \mid \#\left\{i: v^{i}+c^{*} \geq k\right\}=j^{*}\right]=k-c^{*}$. Voters have a dominant strategy to vote yes if and only if $v^{i}+c>k$. Observe that this referendum is constructed so that, in the event $c=c^{*}$, the expected average virtual valuation, conditional on the proportion of yes voters exactly equaling the referendum threshold $j^{*}$, is equal to $k-c^{*}$. Thus, if $c<c^{*}$ this referendum will always fail to produce the public good in the limit, while if $c>c^{*}$ the referendum will always produce the public good in the limit. By the same argument as in the proof of Theorem 2 (see appendix) argument of the previous subsection, welfare losses conditional on $c$ go to 0 for every value of $c$ (in fact, uniformly in $c$ ), except possibly at $c^{*}$. Therefore total welfare losses (integrating over $G(c)$ ) go to 0 for this referendum.

Next consider any $j N$-referendum with $j>j^{*}$. For this $j N$-referendum there

\footnotetext{
${ }^{13}$ Moreover, $j^{*}$ must be strictly between 0 and 1 , since $c^{*}$ is in the interior of the support of $G$, so the $j^{*}$-referendum is not a command mechanism.
} 
exists a critical common value, $c^{* *}>c^{*}$, such that, for large $N$, the public good is only produced when $c>c^{* *}$. Thus, for realizations of $c$ between $c^{*}$ and $c^{* *}$, the public good will inefficiently fail to be produced in the limit, even though the $c$-conditional optimal mechanism requires production in the limit with probability approaching 1. This generates a strictly positive per capita welfare loss, conditional on $c$, on the order of $c-c^{*}$ for realizations of $c$ between $c^{*}$ and $c^{* *}$. Thus, integrating over all possible values of $c$, the expected per capita welfare loss is strictly positive in the limit for any sequence of $j N$-referenda with $j>j^{*}$, and therefore the total welfare loss diverges to infinity. A similar argument applies for any sequence of $j N$ - referenda with $j<j^{*}$. Thus, other simple voting mechanisms are asymptotically inferior to $j^{*}$. QED

Obviously the above proof also shows that the $j^{*} N$-referendum is also uniquely asymptotically optimal in terms of per-capita welfare.

\section{Conclusions}

In an earlier paper, we fully characterized interim efficient public good allocation rules and identified type-contingent tax schemes which implement them under Bayesian Nash equilibrium. In this paper, we proved that for any interim efficient allocation rule there exists a simple dominant-strategy referendum mechanism which approximates the efficiency of that allocation rule in large populations, and uses a minimal message space. In a referendum, individuals simply submit a binary message (a "vote") either for or against production of the public good. If a sufficiently large fraction of the individuals vote in favor, then the public good is provided and the costs are distributed equally in the population. Otherwise, the public good is not 
produced. This provides an approximate "second welfare theorem" for public goods: efficient allocation rules can be (approximately) decentralized by simple voting rules. Moreover, if there is a common value component to the distribution of preferences, then the optimal referendum is unique.

Our results about the asymptotic optimality of referenda were obtained by replicating a population with the same distribution of types. There are a number of interesting extensions, which could generalize the results in this paper in potentially interesting ways. For example, if type-distributions or welfare weights differ across the population, asymptotically optimal referenda might involve asymmetric cost shares, or weighted voting. Other possible directions for future work includes relaxing the assumption of independent types, considering more general forms of common and/or affiliated values, and introducing multidimensional types. 


\section{Appendix}

Proof of Theorem 2: From equations 1 and 2,

$$
\begin{aligned}
\Delta W_{N}^{j^{*}} & =N \int_{\underline{v}}^{\bar{v}} \lambda(v)(v-k)\left(Q_{N}^{o}(v)-Q_{N}^{j^{*}}(v)\right) d F(v) \\
& -N \int_{\underline{v}}^{\bar{v}} \lambda(v)\left(A_{N}^{o}(v)-k Q_{N}^{o}(v)\right) d F(v)
\end{aligned}
$$

Thus, the expected welfare loss is divided into two terms. The magnitude of the first term is on the order of $N$ times the average expected differences in the reduced form production decisions, $Q_{N}^{o}$ and $Q_{N}^{j^{*}}$. The magnitude of the second term is on the order of $N$ times the expected difference between equal cost sharing in the referendum and incentive compatible cost sharing in the optimal mechanism. We apply a central limit theorem below to show that both of these converge to 0 in $N$, although we find that convergence occurs at an order of magnitude faster rate for the second term than the first.

We begin by considering the first term, $N \int_{\underline{v}}^{\bar{v}} \lambda(v)(v-k)\left(Q_{N}^{o}(v)-Q_{N}^{j^{*}}(v)\right) d F(v)$. Recall that both $Q_{N}^{o}$ and $Q_{N}^{j^{*}}$ are deterministic in the limit (i.e., equal either 0 or $1)$. Thus if $j^{*}$ is not chosen so that $Q_{N}^{j^{*}} \approx Q_{N}^{o}$, then we know that the expected welfare loss goes to infinity. However, we know from above that for $j^{*}$ satisfying $E\left[\sum_{i} w_{i} / N \mid \#\left\{i: v^{i} \geq k\right\}=j^{*}\right]=k$ we are guaranteed that $Q_{N}^{j^{*}} \approx Q_{N}^{o}$. Thus, we only need to obtain a rate of convergence to 0 for $Q_{N}^{j^{*}}-Q_{N}^{o}$ and show that this converges to 0 very fast. We show below that the speed of convergence is at least on the order of $\sqrt{N} e^{-N}$, so $N$ times the expected difference in interim quantities converges to 0 , and hence the first term goes to 0 in $N$. 
In the optimal mechanism, ${ }^{14}$ the good is produced if and only if $\sum_{i} w_{i} / N \geq k$. Thus, for an individual with private value equal to $v^{i}$, the interim expected output under the optimal mechanism is simply the probability that the sum of all the other virtual valuations is greater than or equal to $k N-w\left(v^{i}\right)$ which equals the probability that the sample average virtual valuation of the other players is greater than or equal to $\left[k N-w\left(v^{i}\right)\right] /(N-1)$. Denoting the expected value of the virtual valuation of an individual as $\bar{w}$, we know from the Central Limit Theorem that the sample average virtual valuation of $N-1$ has an asymptotically Normal distribution with mean $\bar{w}$ and standard deviation $\sigma_{w} /(N-1)$, where $\sigma_{w}$ is the standard deviation of $w$. Thus, we get

$$
Q_{N}^{o}(v) \rightarrow 1-\Phi\left[\frac{-(\bar{w}-k)-\left(\frac{w(v)-k}{N-1}\right)}{\sigma_{w} /(N-1)}\right]
$$

where $\Phi$ is the cumulative of the unit Normal distribution. Similarly, we can obtain an expression for the asymptotic value of $Q_{N}^{j^{*}}(v)$. It depends only on whether or not $v$ is greater than or less than $k$. Denote by $b(v)$ the vote of an individual of type $v$, which is equal to 1 if $v$ is greater than or equal to $k$ and equals 0 if $v$ is less than $k$. Denote by $\bar{b}$ the ex ante probability of a yes vote (which is simply equal to $1-F(k)$ ), and which also equals the expected fraction of individuals voting yes. Then by a similar argument, we get that

$$
Q_{N}^{j^{*}}(v) \rightarrow 1-\Phi\left[\frac{-\left(\bar{b}-j^{*}\right)-\left(\frac{b(v)-j^{*}}{N-1}\right)}{\sigma_{b} /(N-1)}\right]
$$

\footnotetext{
${ }^{14}$ We ignore the distinction between relaxed optimal and optimal mechanisms for the reasons seen in the proof of Theorem 1.
} 
where $\sigma_{b}$ is the variance of $b$.

By construction of $j^{*}, \lim _{N \rightarrow \infty} Q_{N}^{o}(v)=\lim _{N \rightarrow \infty} Q_{N}^{j^{*}}(v)$. That is, $\bar{b}-j^{*}>0$ if and only if $\bar{w}-k>0$. The difference $Q_{N}^{o}(v)-Q_{N}^{j^{*}}(v)$ converges to

$$
Q_{N}^{o}(v)-Q_{N}^{j^{*}}(v) \approx \frac{1}{\sqrt{2 \Pi}} \int_{A_{N}}^{B_{N}} e^{-x^{2} / 2} d x
$$

where

$$
A_{N}=-\frac{\sqrt{N}\left(\bar{b}-j^{*}\right)}{\sigma_{b}}-\frac{b(v)-j^{*}}{\sigma_{b} \sqrt{N}}
$$

and

$$
B_{N}=-\frac{\sqrt{N}(\bar{w}-k)}{\sigma_{w}}-\frac{w(v)-k}{\sigma_{w} \sqrt{N}}
$$

Without loss of generality, assume that $\frac{\bar{b}-j^{*}}{\sigma_{b}}>\frac{\bar{w}-k}{\sigma_{w}}$, so that, for sufficiently large $N$, $A_{N}<B_{N}$. Then for large $N$,

$$
Q_{N}^{o}(v)-Q_{N}^{j^{*}}(v) \approx N^{1 / 2}\left(\frac{\bar{b}-j^{*}}{\sigma_{b}}-\frac{\bar{w}-k}{\sigma_{w}}\right) \frac{1}{\sqrt{2 \Pi}} e^{-N\left(\frac{\bar{w}-k}{\sigma_{w}}\right)^{2}}
$$

Therefore, the expected difference between the interim expected quantities under the optimal mechanism and the $j^{*}$ mechanism, $N\left(Q_{N}^{o}(v)-Q_{N}^{j^{*}}(v)\right)$, is on the order of $N^{3 / 2} e^{-N}$, which converges to 0 in $N$. This establishes that the first term of the expression for the total surplus loss goes to 0 .

The second term of that expression is

$$
N \int_{\underline{v}}^{\bar{v}} \lambda(v)\left(A_{N}^{o}(v)-k Q_{N}^{o}(v)\right) d F(v)
$$


This can be rewritten as

$$
\int_{\underline{v}}^{\bar{v}} \lambda(v)\left[N\left(A_{N}^{o}(v)-\bar{A}\right)-N k\left(Q_{N}^{o}(v)-\bar{Q}\right)\right] d F(v)
$$

which can be further broken down into two terms:

$$
\int_{\underline{v}}^{\bar{v}} \lambda(v) N\left(A_{N}^{o}(v)-\bar{A}\right) d F(v)
$$

and

$$
\int_{\underline{v}}^{\bar{v}} \lambda(v) N k\left(Q_{N}^{o}(v)-\bar{Q}\right) d F(v) .
$$

Consider the second of these terms. Because $\lambda(v)$ is bounded, we just need to show that

$$
\left.\int_{\underline{v}}^{\bar{v}} N \mid Q_{N}^{o}(v)-\bar{Q}\right) \mid d F(v) \rightarrow 0
$$

The expression $\left.\mid Q_{N}^{o}(v)-\bar{Q}\right) \mid$ is less than or equal to $Q_{N}^{o}(\bar{v})-Q_{N}^{o}(\underline{v})$, so we only need to show that

$$
\int_{\underline{v}}^{\bar{v}} N\left[Q_{N}^{o}(\bar{v})-Q_{N}^{o}(\underline{v})\right] d F(v)=N\left[Q_{N}^{o}(\bar{v})-Q_{N}^{o}(\underline{v})\right] \rightarrow 0
$$

Recall that $Q_{N}^{o}(v)=\operatorname{prob}\left\{\bar{w}-\frac{k}{N-1} \geq \frac{w(v)}{N-1}\right\}$ so, using an argument similar to the one above, the difference $Q_{N}^{o}(\bar{v})-Q_{N}^{o}(\underline{v})$ converges to

$$
Q_{N}^{o}(\bar{v})-Q_{N}^{o}(\underline{v}) \approx \frac{1}{\sqrt{2 \Pi}} \int_{A_{N}}^{B_{N}} e^{-x^{2} / 2} d x
$$


where

$$
A_{N}=-\frac{\sqrt{N}(\bar{w}-k)}{\sigma_{w}}-\frac{w(\underline{v})-k}{\sigma_{w} \sqrt{N}}
$$

and

$$
B_{N}=-\frac{\sqrt{N}(\bar{w}-k)}{\sigma_{w}}-\frac{w(\bar{v})-k}{\sigma_{w} \sqrt{N}} .
$$

This is on the order of $\sqrt{N} e^{-N}$, so $N\left[Q_{N}^{o}(\bar{v})-Q_{N}^{o}(\underline{v})\right] \rightarrow 0$ as $N \rightarrow \infty$. Therefore

$$
\int_{\underline{v}}^{\bar{v}} \lambda(v) N k\left(Q_{N}^{o}(v)-\bar{Q}\right) d F(v) \rightarrow 0
$$

as desired. By incentive compatibility, $A^{\prime}=v Q^{\prime}$, and by assumption $v<\bar{v}<\infty$, so it also follows that

$$
\int_{\underline{v}}^{\bar{v}} \lambda(v) N\left(A_{N}^{o}(v)-\bar{A}\right) d F(v) \rightarrow 0
$$

Thus $\lim _{N \rightarrow \infty} \Delta W_{N}^{j^{*}} \rightarrow 0$. QED 


\section{REFERENCES}

Al-Najjar, Nabil and Rann Smorodinsky, 2000. "Pivotal Players and the Characterization of Influence," Journal of Economic Theory, 92, 2, 318-42.

d'Aspremont, Claude and L.-A. Gerard-Varet, 1979. "Incentives and Incomplete Information," Journal of Public Economics, 11, 25-45.

Guesnerie, Roger and Jean-Jacques Laffont, 1984. "A Complete Solution to a Class of Principal Agent Problems with an Application to the Control of a Self-Managed Firm," Journal of Public Economics, 25: 329-69.

Hurwicz, Leonid, 1972. "On Informationally Decentralized Systems," in Radner, R. and McGuire. C. (eds.), Decision and Organization. North Holland, Amsterdam, pp. 297-336.

Ledyard, John O. and Thomas R. Palfrey, 1994. "Voting and Lottery Drafts as Efficient Public Goods Mechanisms," Review of Economic Studies, 61, 327-55.

Ledyard, John O. and Thomas R. Palfrey, 1999. "A Characterization of Interim Efficiency with Public Goods," Econometrica, 67, 435-48.

Mailath, George and Andrew Postlewaite, 1990. "Asymmetric Information Bargaining Problems with Many Agents," Review of Economic Studies, 57, 351-67.

Marschak, Jacob and Roy Radner, 1972. Economic Theory of Teams, Yale University Press, New Haven.

Mookherjee, Dilip and Stefan Reichelstein, 1982. Journal of Economic Theory, 56, $2,378-99$.

Mount, K. and Stanley Reiter, 1974. "The Informational Size of Message Spaces," Journal of Economic Theory, 8, 161-92. 